ActA Poetica 361

enero-junio

$2015(71-85)$

\title{
J. M. Coetzee: en el callejón de Samuel Beckett
}

\section{J.M. Coetzee: in the alley of Samuel Beckett}

Diego SheinbAum

El autor muestra la influencia de Samuel Beckett en la obra del escritor sudafricano. Analiza las dos primeras novelas de J. M. Coetzee, Dusklands (1974) e In the Hearth of the Country (1977), para explicar cómo sus protagonistas comparten el solipsismo de los personajes becketianos; sin embargo, su destino cambia bajo la lógica del Colonialismo. A partir de este grado cero de la escritura, los personajes del novelista sudafricano inician un viaje que, en términos filosóficos, va de la parodia del ego cartesiano hacia el horizonte de reconocimiento hegeliano.

PAlAbrAS clAve : Coetzee, Beckett, colonialismo, ego cartesiano, reconocimiento hegeliano.

The author shows the influence of Samuel Beckett's work in the oeuvre of the South African writer. He analyzes the first two novels of J. M. Coetzee, Dusklands (1974) and In the Hearth of the Country (1977) in order to explain how their protagonists share the solipsism of Beckett's characters; however, their fate changes under the logic of colonialism. From this zero degree of writing, the characters of the South African novelist begin a journey that, in philosophical terms, takes them from the Cartesian ego towards the horizon of Hegelian recognition.

keyworDs : Coetzee, Beckett, colonialism, Cartesian ego, Hegelian recognition.

Fecha de recepción: 17 de junio de 2014

Fecha de aceptación: 11 de agosto de 2014 



\author{
Diego SheinbAum \\ Universidad Iberoamericana \\ drorian@gmail.com
}

\title{
J. M. Coetzee: en el callejón de Samuel Beckett
}

Para iniciarse en la obra de Coetzee es suficiente vaciar un sobre de harina en un vaso de agua. Con ello podemos repetir en la imaginación el experimento que utiliza Viktor Shklovsky para explicar el fenómeno de la creatividad. Las partículas de harina parecen suspendidas, pero pronto se puede percibir cómo empiezan a vibrar, cómo están sujetas y responden a billones de impulsos invisibles. De la misma manera, nos dice Shklovsky, el arte no lo crea una sola voluntad, un solo genio. El creador individual sólo es el locus geométrico de líneas que se intersectan, de fuerzas que nacen fuera de su conciencia (Shklovsky: 27). La obra de Coetzee evidencia dos fuerzas notorias, dos vectores que chocan produciendo efectos inesperados. Por una parte, la herencia modernista, la reescritura de sus lenguajes, motivos, temas y actitudes. Coetzee hace explícita esta deuda en una conferencia titulada "Homage"; en ella explica que no fueron los escritores sudafricanos quienes le enseñaron a sentir y a responder a la situación en su país, sino Rilke y Musil, Ford y Pound, Zbiniew Herbert y Beckett; es decir, una pléyade de escritores modernistas. ${ }^{1}$ La otra gran fuerza es la lógica de los regímenes coloniales e imperiales, la cual se torna en toda una exploración psicológica, ética y política dentro de su obra. En esa constelación

1 J. M. Coetzee, “Homage”, en The Three Penny Review, núm. 53 (primavera, 1993). 
modernista, Beckett se estremece con particular fuerza y vale la pena esbozar la manera en que su obra colisiona con la lógica colonial en las primeras novelas de Coetzee. Vale la pena esbozar la manera en que la obra de Beckett colisiona con la lógica colonial, presente en la escritura de Coetzee, para entender la singular forma que toma este choque en relación a Kafka.

El goce y análisis de la obra de Beckett han acompañado a Coetzee a lo largo de su carrera, desde su disertación doctoral, The English Fiction of Samuel Beckett: An Essay in Stylistic Analysis, hasta el ensayo literario presentado en 2008 en una conferencia en Japón, "Eight Ways of Looking at Samuel Beckett". ${ }^{2}$ En sus ficciones se advierte esta influencia, sobre todo, en sus primeras dos novelas. En ellas la vida bajo el colonialismo es abordada en tres momentos de su historia: en los inicios, en su mediodía y en los años de colapso del Imperio (Pippin: 1941). En ellas comienza el recorrido que seguirá realizando Coetzee en cada una de sus novelas pertenecientes al ciclo del Apartheid. Se trata de un viaje desde el encierro en el monólogo del ego hasta el intento de reconocer otras voces, otras conciencias, con el afán de ir más allá de la negación y la violencia. En términos filosóficos es un viaje que va desde el polo de la parodia cartesiana hacia el drama del reconocimiento hegeliano. Este reconocimiento será el horizonte hacia el cual se moverán cada uno de sus protagonistas en las novelas de los ochenta, mostrando que las rutas del autoengaño y de la autocomplacencia tienen graves consecuencias psicológicas (Pippin: 19-41).

La primera novela de Coetzee, Dusklands (1974), encarna el grado cero de esta trayectoria. El texto está conformado por dos ficciones, la

${ }^{2}$ La lista completa de los trabajos de Coetzee sobre Beckett incluye: The English Fiction of Samuel Beckett: An Essay in Stylistic Analysis, Ph.D. dissertation, University of Texas at Austin, 1969; "Samuel Beckett and the Temptations of Style", Doubling the Point, Essays and Interviews, ed. David Atwell Harvard University Press, Cambridge, 1992; "Samuel Beckett's Lessness: An Exercise in Decomposition", Computer and the Humanities 7 núm. 4, 1973, 195-98; "Introduction to Samuel Beckett", The Grove Centenary Edition vol. 4, Grove Press, Nueva York, 2006; "Samuel Beckett in Cape Town: An Imaginary History", Beckett Remembering, Bloomsbury, Londres, 2006, 74-77; "Eight Ways of Looking at Samuel Beckett", Borderless Beckett (Amsterdam: Rodopi, 2008); "The Making of Samuel Beckett", New York Review of Books 56, 7, April 30, 2009. 
primera situada en Estados Unidos en el contexto de la guerra de Vietnam, y la segunda situada en África en el contexto de los viajes de exploración del sigloxviii . Estas narraciones están vinculadas por la historia del colonialismo y también por lo que el autor sudafricano llamó, en relación a Beckett, la comedia del punto de vista: el colapso de la ilusión de objetividad a través del juego de confusiones entre autor, narrador y personaje. ${ }^{3}$ La primera de estas ficciones, "El proyecto Vietnam", narra el desplome mental de Eugene Dawns, un hombre racional, formado en la teoría de juegos, que ama la disciplina, está obsesionado por el pensamiento estratégico y todo lo ve en términos de lucha de poderes. En el repertorio de los protagonistas de Coetzee, Dawns representa un claro extremo: encarna la negación de cualquier empatía por el otro, el encierro en las propias maquinaciones, la elaboración de un monólogo impermeable a voces ajenas. Sus pensamientos paranoicos lo alejan de nosotros. Al parecer estamos ante la desquiciada parodia de un fascista que admira el poder, que sólo quiere reconocer la parte fuerte de las personas y desprecia lo débil, lo servicial, lo amoroso. Sin embargo, poco a poco, el lejano testimonio se nos revela como un monólogo mucho más cercano, quizá el discurso subterráneo, no reconocido, de nuestra civilización occidental. Por eso, posiblemente, la personalidad y acciones de Dawns nos repelen con tanta fuerza. Su discurso articula aquello que no deseamos escuchar, para cuyo olvido hacemos todo. Nos muestra el retrato del hombre occidental que no repara en que, detrás de su racionalidad, hay simplemente la voluntad de controlar, dominar, disciplinar o destrozar todo lo que es diferente a él.

Esta voluntad se expresa en la relación de Eugene con su cuerpo, su familia y la naturaleza. Si las novelas de Beckett pueden verse como parodias del ego cartesiano, dolorosas comedias de un yo encerrado en un cuerpo y en un mundo ajeno, hombres que viven en la duda y la

${ }^{3}$ J. M. Coetzee, Doubling the Point, Essays and Interviews, ed. David Atwell, Harvard University Press, Cambridge, 1992. En la primera narración, titulada El proyecto Vietnam, el jefe del protagonista se llama Coetzee, mientras que en la segunda, titulada La narración de Jacobus Coetzee, se multiplican los lazos de identidad: tenemos al lejano antepasado y explorador Jacobus Coetzee; tenemos al Dr. S. J. Coetzee, académico de la Universidad de Stellebosch, primer recopilador del texto; y tenemos al supuesto autor J. M. Coetzee que ha realizado una nueva traducción y ha ordenado el material en un nuevo orden. 
incertidumbre sobre los objetos más cercanos, a los que intentan comprender a través de inventarios, conmutaciones y cálculos estrafalarios, esta primera novela de Coetzee hace explícita dicha alusión. En el informe que escribe Dawns, cuya introducción leemos, se establece que, a diferencia de los occidentales, los vietnamitas son ajenos a "the rational promptings of self-interest" y que las trasmisiones por radio han fracasado porque utilizan "the voice of René Descartes" (Coetzee 1974: 28). ${ }^{4}$ Pero mucho más relevante es el vínculo que se establece entre el ego cartesiano y el colonialismo, que no se limita a la participación en el proyecto Vietnam y se manifiesta, sobre todo, en el impulso aventurero que nuestra civilización festeja hasta en la publicidad de autos: "I have an exploring temperament. Had I lived two hundred years ago I would have had a continent to explore, to map, to open to colonization" (31-32). ${ }^{5}$ Este afán de conquistar nuevos territorios se despliega hacia afuera y hacia dentro: "I approve of the enterprise of exploring the self. I am deeply interested in myself. I should like to see in black and white an explanation of this disturbed and disturbing act of mine" (46). Dawns representa la mentalidad de nuestro pasado y, como su nombre lo sugiere, del futuro hacia el cual nos dirigimos, un futuro sustentado en el mito ya no de la madre Tierra, sino del ser humano que se produce a sí mismo, que nace de su propia cabeza, a través de la tecnología, como la diosa Atenea de la cabeza de Zeus: "Is it not time that the earthmother be supplanted by her own faithful daughter, shaped without woman's part? The age of Athene dawns" (26)?

Mientras que el destino de Eugene muestra las consecuencias psicológicas de negar esa otredad que culmina en la paranoia y el encierro, la segunda mitad de Dusklands — "The Narrative of Jacobus Coetzee"-

4 "Los apuntes racionales del interés propio. [...] La voz de René Descartes" (Coetzee 2009: 38).

5 "Tengo un temperamento de explorador. De haber vivido hace doscientos años, habría tenido un continente entero para explotar, cartografiar y abrir a la colonización" (Coetzee 2009: 53).

6 "Apruebo la empresa de explorarse a uno mismo. Me interesa mucho mi propio yo. Me gustaría ver una explicación por escrito de este acto mío perturbado y perturbador" (Coetzee 2009: 72).

7 “ ¿Acaso no es hora de la que la madre-tierra sea suplantada por su fiel hija, engendrada sin participación de mujer? Así amanece la era de Atenea" (Coetzee 2009: 46). 
empieza en el otro extremo, en las grandes extensiones africanas, en los primeros viajes de exploración. La mirada ahora apunta hacia afuera. Sin embargo, pronto nos damos cuenta que es una mirada para determinar y trazar diferencias. El interés en el otro es un puro interés personal para hacerse de sus riquezas y territorios. El otro está afuera para ser invadido, poseído y, sobre todo, negado. Los ojos que miran son los de un cazador. La descripción de los hotentotes que realiza Jacobus en las primeras páginas se convierte rápidamente en un manual de cómo cazar bosquimanos (una práctica muy cristiana, uno supone): "the only way to kill a Bushman is to catch him in the open where your horse can run him down" (58). ${ }^{8}$ Estas observaciones preludian el viaje que Jacobus realiza más allá del Gran Río, para encontrarse con los namaquas. En ellas va surgiendo lo que se convertirá en el monólogo de un "domador de lo salvaje", una reflexión sobre la vida en esas largas extensiones, sobre la vida sin límites, que Jacobus desarrollará, curiosamente, en medio de alucinaciones provocadas por una infección intestinal:

Only the eyes have power. The eyes are free, they reach out to the horizon all around. Nothing is hidden from the eyes. [...] I become a spherical reflecting eye moving through the wilderness and ingesting it. [...] What is there that is not me? I am a transparent sac with a black core full of images and a gun (79). ${ }^{9}$

El ojo armado con una pistola no es una imagen muy distante de la experiencia cotidiana de un chico jugando con un videojuego, o un militar conduciendo una guerra en este nuevo milenio. El ojo cartesiano no necesita un evangelio de la compasión sino un evangelio de la pistola para tratar con lo otro. ${ }^{10}$ Este poder sobre la vida y la muerte es una face-

8 "La única manera segura de matar a un bosquimano es atraparlo en campo abierto, donde tu caballo lo pueda alcanzar" (Coetzee 2009: 85).

9 "Sola la mirada tiene poder. La mirada es libre, se extiende por el horizonte en todas direcciones. Nada se oculta a la mirada. [...] Me convierto en un ojo reflejante esférico que se mueve por el yermo y lo ingiere [...]. ¿Qué existe que no sea parte de mí? Soy un saco transparente con un núcleo negro lleno de imágenes y un arma de fuego" (Coetzee 2009: 112-113).

10 "The gun stands for the hope that there exists that which is other than oneself. The gun is our last defense against isolation [...], is our mediator with the world and therefore our savior $[. .$.$] . The gun saves us from the fear that all life is within us" (Coetzee$ 
ta del ser humano en tanto hombre-Dios. Como un entrepreneur, Jacobus se atribuye todos los esfuerzos y los éxitos de la expedición. ${ }^{11}$ Esta elevada concepción de sí mismo, en tanto voluntad triunfante, se volverá asesina cuando los namaquas no le dan el reconocimiento que espera. Víctima de una enfermedad gastrointestinal, Jacobus se ve recluido en una de las chozas reservadas para la menstruación de las mujeres. Durante días lucha con un furúnculo que le ha salido en la nalga hasta que logra abandonar la aldea en compañía de su fiel sirviente Klawer. Pero Jacobus realiza una segunda expedición, esta vez con el claro propósito de exterminar la aldea. Así resuelve su relación con los otros.

La primera novela de Coetzee guarda reminiscencias con Molloy, pieza constituida también por dos ficciones separadas por un boquete. Con este libro, Beckett inicia un recorrido formal y emocional que lo llevará a purgar las convenciones de la novela tradicional y "cavar su propia tumba en prosa" (Alvarez: 54). En su trilogía el autor irlandés se desprende progresivamente de los artificios esenciales del gremio: desdibuja historias, personajes y entornos. Molloy pierde progresivamente la capacidad de moverse, sus piernas dejan de funcionarle, para avanzar tiene que arrastrarse hasta quedar varado en una zanja. La ciudad, el campo, el mar y el bosque, que apenas están esbozados, desaparecen para la segunda novela. El nuevo héroe, Malone, es un octogenario postrado en su cama. Las historias que escribe se van agotando. Para la tercera entrega el héroe ya no tiene nombre, no tiene rasgos personales, ni siquiera un cuerpo. Lo único que le queda es una voz, formada por palabras que no son suyas.

Molloy, Malone Dies y The Unnamable forman un corredor de la muerte (33-85). La gesta creativa de Beckett consiste en llevarnos a

1974: 79). "El arma de fuego representa la esperanza de que exista algo que no sea uno mismo. El arma de fuego es nuestra última defensa contra el aislamiento [...], es nuestra mediadora con el mundo y por tanto nuestra salvadora [...]. El arma de fuego nos salva del miedo de que toda la vida esté dentro de nosotros" (Coetzee 2009: 113).

11 "My Hottentos and my oxen had given me faithful service: but the success of the expedition had flown from my own enterprise and exertions. [...] They saw me as their father. They would have died without me" (Coetzee 1974: 64). "Mis hotentotes y mis bueyes me habían servido con fidelidad. Pero el éxito de la expedición había venido de mi propia iniciativa y mis propios esfuerzos [...]. Ellos me veían como a un padre. Se habrían muerto sin mí" (Coetzee 2009: 92). 
este fondo de oscuridad, decadencia y depresión humana a través de un estilo translúcido, ordenado y conciso. Cientos de pequeñas y livianas frases se alternan. Las afirmaciones dan paso a correcciones o negaciones, de tal manera que la superficie reverbera como las crestas de un mar convulso a mitad de la tarde. "The island, that's all the earth I know. I don't know it either, never having the stomach to look at it" (Beckett 2009: 320). ${ }^{12}$ La linealidad de la lógica se multiplica en varias direcciones y transparenta la turbulencia. Los trazos regresan sobre sí mismos y logran que la representación palpite. Beckett arrastra los sustantivos impolutos de la filosofía a su fondo corporal: "But let me complete my views before I shit on them" (331).' Las disertaciones adquieren la proximidad pegajosa de nuestros fluidos y el ritmo de nuestro metabolismo: "And in no time I'll be a network of fistulae, bubbling with the blessed pus of reason" (347). ${ }^{14}$ Los procesos mentales se sienten como castigos corporales: "They have inflected the notion of time on me" (319). ${ }^{15}$ Así, el escritor irlandés convierte los monólogos de la filosofía en un repertorio cómico. Personajes, entorno y estilo nos hacen sentir atrapados en medio del tedio, la desolación y del sufrimiento, en una especie de purgatorio secular, salpicado por un cruel humorismo.

A pesar de la desnudez, a pesar de que Beckett se ha desembarazado de todos los artificios que volvían atractiva la novela al público, la voz de sus monólogos nos mantiene cautivos, y nos deslizamos por los fríos mares de su depresión terminal como marineros que sólo escuchan el canto de las sirenas: "Then they uncorked the champagne. One of us at last! Green with anguish! A real little terrestrial! Choking in the chlorophyll! Hugging the slaughter-house walls!" (309-310). ${ }^{16}$

12 "Lo único que conozco es la isla, nada más. Y tampoco la conozco, pues nunca tuve la fuerza para mirarla" (Beckett 1998: 80). La traducción pierde el énfasis corporal y cómico de la frase final.

13 "Pero concluyamos nuestro pensamiento antes de ensuciar encima" (Beckett 1998: 93). La traducción es torpe y pierde el alto y cómico contraste de Beckett.

14 "Y pronto no seré más que una red de fístulas acarreando el pus bienhechor de la razón" (Beckett 1998: 112). Una traducción más certera culminaría en: "la bendita pus de la razón".

15 "Si también ellos me infligieron la noción de tiempo" (Beckett 1998: 78).

16 "Entonces ellos descorcharon el champaña: ¡Es de los nuestros! ¡Verduzco de angustia! ¡Un verdadero terrícola! ¡Ahogado en clorofila! ¡Rozando los mataderos!” 
Si bien en sus primeras novelas Coetzee está lejos de lograr ese hipnotismo en la prosa, la escritura modernista vuelve a sentirse en su segunda obra, In the Heart of the Country. En ella, como lo percibe Gilbert Yeoh, Coetzee trasplanta el paradigma becketiano a la realidad sudafricana. La problemática metafísica y epistemológica del escritor irlandés se transforma al tocar suelo africano en los dilemas éticos y políticos de los protagonistas de Coetzee. La alienación existencial se vuelve marginación social; incluso, el enigma filosófico de la escritura que logra alzarse sobre la nada y el silencio, se convierte en la necesidad de responder a los horrores y, también, el impulso contrario de sentirse culpable por ser blanco, sin la autoridad para hablar de las injusticias (Yeoh: 117-137).

Las marcas de la obra de Beckett se extienden como manchas de café por las páginas de Coetzee. En esta segunda obra encontramos de nuevo el monólogo obsesivo del ego, el encierro solipsista del protagonista y el cuestionamiento de sus propias representaciones, a veces hasta alcanzar el auto-sabotaje. Como el héroe de Malone Dies, Magda pasa buena parte de su vida en un cuarto: "I am the one who stays in her room reading or writing or fighting migraines" (Coetzee 1977: 1).17 A diferencia de los protagonistas de Beckett, sin embargo, ella no vive fuera del tiempo histórico: "The colonies are full of girls like that, but none, I think, so extreme as I" (1). ${ }^{18}$ La historia del colonialismo que se tiende como una enorme cúpula sobre América, Asia y África es vivida por Magda y los futuros protagonistas de Coetzee como el confinamiento en una habitación cerrada. En las extensiones africanas, en la historia que los escribe como colonizadores, en el lenguaje de poder y de jerarquía que heredan de sus padres, se sentirán tan oprimidos como los vagabundos de Beckett entre cuatro paredes:

I cannot carry on with these idiot dialogues. The language that should pass between me and these people was subverted by my father and can-

(Beckett 1998: 67). Una traducción más exacta sería: “¡Abrazando las paredes del matadero!"

17 “A mí me toca quedarme en la habitación de ella, leyendo o combatiendo contra las migrañas" (Coetzee 2003: 7).

18 "Las colonias están repletas de muchachas así, aunque no creo que haya ninguna tan extrema como yo" (Coetzee 2003: 1). 
not be recovered. What passes between us now is a parody. I was born into a language of hierarchy, of distance and perspective. It was my father-tongue (97). ${ }^{19}$

Magda está consciente de los límites de su habla; esa conciencia es posible porque Coetzee se levanta con un pie en el furor modernista y con el otro sobre la lógica del colonialismo. Este movimiento simultáneo se volverá particularmente claro en el homenaje que hace a Kafka en su cuarta novela, Life and Times of Michael $K$, muestra prístina de un fabulador que defiende la autonomía de sus ficciones y, al mismo tiempo, las sitúa en un contexto más amplio que invariablemente produce resonancias éticas y políticas. Esta combinación hace de Coetzee un escritor singular, un profesor de literatura moderna que recupera ficciones conocidas y las tiñe, las actualiza, tras sumergirlas en la cubeta de la historia, la cual, como sabemos, contiene todo menos agua cristalina (cfr. Huchteon).

Las limitaciones del discurso de Magda son las limitaciones que comparten el sujeto de las ficciones modernistas y el sujeto construido por la lógica imperialista. ${ }^{20}$ Lo interesante, como lo señala Robert Pippin, es que las ficciones de Coetzee, más que historias moralistas, muestran las carencias, muchas veces insostenibles, de ambas actitudes. El encierro en el ego termina por engendrar un sujeto fallido, fantasmagórico, que si bien tiene la habilidad para auto-constituirse no es visto ni reconocido por la realidad social (19-41).

Las consecuencias de vivir bajo la lógica del colonialismo, sin otro que pueda darnos un reconocimiento genuino, hacen que el monólogo de Magda se tambalee entre una frecuente interrogación y una no

19 "No puedo mantener por más tiempo estos diálogos besugos. El lenguaje que debería trasmitirse entre estas personas y yo lo subvirtió por completo mi padre; ya no es posible recuperarlo. Lo que ahora pasa entre unos y otros es pura parodia. Nací para desembarcar en un lenguaje de jerarquía, de distancia, de perspectiva. Era la lengua de mi padre" (Coetzee 2003: 134). El traductor selecciona mal la palabra "besugos" en vez de simplemente "idiotas"; lo mismo con "desembarque" cuando la traducción natural sería "nací".

20 "It is possible that I am a prisoner not of the lonely farmhouse and the stone desert but of my stony monologue?” (Coetzee 1977: 12). “Es posible que sea yo prisionera no solo de la granja solitaria y del desierto de pedregales, sino también de mi pétreo monólogo?" (Coetzee 2003: 22). 
menos frecuente y mucho más errática fabulación. La narración sufre a cada paso de brotes fantasmagóricos. Las versiones de cuanto sucedió se multiplican hasta la contradicción. Magda asesina dos veces a su padre $y$, al final, lo pasea en una silla de ruedas por la baranda. Esta profusión de historias termina por sabotear la representación. Con ello Coetzee vuelve a hacer un gesto hacia dentro y hacia afuera del vanguardismo de Beckett, cuyo virtuosismo radica, probablemente, en la intermitencia que logra entre ilusionismo y anti-ilusionismo. Este virtuosismo se debe en gran medida a que antes de realizar esta operación, Beckett reduce al mínimo el material para la representación y, sin embargo, el efecto de las ilusiones es igual o más poderoso. En vez de decenas de personajes, de tramas con saltos, volteretas y descubrimientos, de las varias residencias y los miles de objetos descritos por las novelas del siglo xix , tenemos protagonistas casi únicos, lisiados, que se arrastran o están encerrados en cuartos, que no conviven con otros seres humanos, sino que limitan su contacto a unos cuantos objetos nimios. Es como si la energía creativa derrochada por la tradición novelística se hubiera agotado, y los personajes de Beckett ya ni siquiera tuvieran la fuerza para mover sus piernas o levantar sus párpados. O como si el desencantamiento del mundo articulado por Descartes volviera hasta las propias narraciones una res extensa sin alma, sin espíritu. Y, sin embargo, en esta profunda oscuridad, en esta pobreza, los protagonistas de Beckett logran encender ilusiones como si frotaran un cerillo. En la noche cerrada su luz se vuelve más deslumbrante; pero es una flama vacilante, que constantemente revira, se corrige a sí misma, se contradice y amenaza con extinguirse.

Coetzee no es tan extremo ni en la intermitencia ni en la reducción del foco y, sin embargo, en su obra se siente también el minimalismo de Beckett. Sus novelas presentan un reducido elenco de personajes, los cuales ocupan de manera flagrante sólo una esquina de la vasta realidad. La voz narrativa no se permite declaraciones sentenciosas o universales ni saltos por varias conciencias. Más bien es un murmullo íntimo y desalmado, tan sutil en su exploración interior como duro y frío en su juicio de sí mismo. ${ }^{21}$ Esta reducción del foco se puede sentir también

${ }^{21}$ En las novelas de su etapa de madurez como Age of Iron, The Master of Petersburg y Disgrace esto se torna en un magistral uso de la tercera persona. Para muchos 
en las tramas de sus novelas. La arquitectura dramática se diluye en una serie de variaciones sin una estructura climática. En ellas se siente la necesidad de mantenerse abierto al flujo de la vida y al flujo de las interpretaciones. Se siente el compromiso modernista con la experiencia, algo cada vez más ajeno a una sociedad del entretenimiento, acostumbrada a diseñar sus historias de acuerdo al paladar de los espectadoreš?

Si bien Patrick Hayes ha señalado que la prosa de Coetzee se sitúa de manera desestabilizadora entre el realismo y el anti-realismo manteniendo un potencial anti-fundacional (cfr. Hayes), hay una fina diferencia respecto de Beckett. Mientras que el escritor irlandés recorre el callejón donde la representación se termina, golpeándose una y otra vez contra este último límite, Coetzee se da la vuelta y regresa a la tradición de la novela moderna, para combinar los dos linajes opuestos sobre los cuales ha llamado la atención Robert Stam. Por un lado, la novela paródica, intertextual y mágica de Cervantes, la cual exhibe y denuncia su propio artificio. Por otro, la novela documental de Defoe que intenta crear un fuerte efecto de realidad y ocultar su factura literaria (Stam: 1). De esta manera, en sus novelas el ilusionismo coexiste con la reflexividad, la representación es, también, parodia y diálogo con otros

escritores esta elección sería una cuestión puramente estética, pero en la obra de Coetzee el artificio cobra resonancias morales y filosóficas. Esto se debe en gran medida a la peculiar manera de moldear a su protagonista y a los otros personajes. Con la narración indirecta en tercera persona, Coetzee logra una verdadera integración de dos puntos de vista en su protagonista, un yo en un él, lo cual se complementa por la forma en que está concebida la relación con los otros personajes, quienes en la medida en que son observados desde esta subjetividad juiciosa parecen menos creaciones independientes y externas, que centros de confrontación, núcleos de preguntas y, sobre todo, de opacidades, demasiado cerca del protagonista para no sentirse definido por ellos, demasiado lejos para agotar el conocimiento que tiene de ellos. Estas dos operaciones, la integración de dos puntos de vista y el reconocimiento de que uno sólo es una persona entre otras, coinciden con lo que Thomas Nagel definió como las fuerzas esenciales detrás del desarrollo de una posición moral (cfr. Nagel).

${ }^{22}$ En las novelas de madurez la unidad dramática se consolida alrededor del párrafo compacto. A veces en el espacio de seis líneas tenemos la oposición y el drama de una obra de tres actos. No sólo se trata de que las sensaciones, percepciones y reflexiones aparezcan diáfanas y entrelazadas a otra conciencia, sino que los pronombres funcionan como articulaciones que dan movimiento y permiten la oposición entre las dos conciencias. Gracias a ello los párrafos cobran la forma traslúcida, profunda y tensa de pequeños cubos de hielo. 
autores. Esta reflexividad se muestra en el continuo cuestionamiento que realizan los protagonistas sobre sus representaciones. Ese aspecto se volverá más notorio en novelas posteriores; en ellas Coetzee avanza desnudando la elaboración de tal manera que la obra adquiere el candor de los bocetos de pintura, donde nos sentimos dentro del proceso de la creación y no enfrentados a una obra terminada y, quizá por eso, muerta. Las novelas se convierten en aventuras por encontrar un significado, configuraciones tan provisionales al principio como después del punto final. Si bien en las novelas de Beckett encontramos comentarios y reacciones ante lo escrito que aumentan el efecto cómico, en las ficciones de Coetzee esto crece hasta el punto que sus novelas no sólo parecen desarrollarse a partir de borrar la frontera entre creación y evaluación crítica, sino que parecen armadas para contrarrestar las críticas y las interpretaciones posteriores. Cada una de ellas surge ante el público sólo cuando ha logrado hacerse, como Flaubert exigía, de una brillante armadura.

Pero la interrogación en torno a la propia obra no se queda, como en mucho del postmodernismo, en el juego narcisista de sólo hablar de uno mismo, de sólo hacer referencia al propio texto. A partir de In the Heart of the Country, los protagonistas de Coetzee intentan enlazar los deseos de este sujeto modernista o imperialista, que hemos heredado y nos han impuesto las condiciones hegemónicas, con las aspiraciones que nacen de un reconocimiento mutuo y de un horizonte de justicia. Estas tentativas acaban la mayoría de las veces en el fracaso, pero no sin antes exhibir las limitaciones sociopolíticas del discurso. Aunque Magda comparte la casa, el trabajo e incluso el sexo con la joven pareja de sirvientes no logra poner un pie fuera de la lógica colonial de sus relaciones: "I cannot say whether Hendrik and Anna are guests or invaders or prisoners" (Coetzee 1977: 112). ${ }^{23}$ Sin embargo, ya aquí las conjunciones disyuntivas logran explorar representaciones alternativas del otro, y seguir con escepticismo los caminos del deseo. ${ }^{24}$ Lo que es

23 "No sabría decir si Hendrik y Anna son invitados, invasores o prisioneros" (Coetzee 2003: 154).

24 "To the spur of desire we have only one response: to capture, to enclose, to hold. But how real is our possession?" (Coetzee 1977: 114). "A la espuela del deseo sola- 
un gesto en esta segunda novela se convertirá en toda una peripecia en su siguiente obra, Waiting for the Barbarians.

\author{
bibliogrAfíA
}

Alvarez , A . Beckett. London: Fontana Press, 1973.

beckett , SAmuel . El innombrable. Trad. R. Santos Torroella. Barcelona: Alianza, 1998.

beckett , Samuel . Three Novels. Molloy, Malone Dies, The Unnamable. Nueva York: Grove Press, 2009.

Coetzee , J. m.Dusklands. Nueva York: Penguin Books, 1974.

C oetzee , J. m.In the Heart of the Country. Nueva York: Penguin Books, 1977.

Coetzee , J. m Doubling the Point, Essays and Interviews. Ed. David Atwell.

Cambridge: Harvard University Press, 1992.

Coetzee , J. m. "Homage", en The Three Penny Review, núm. 53 (primavera 1993).

C oetzee , J. m.En medio de ninguna parte. Trad. Miguel Martínez-Lague. Barcelona: Random House Mondadori, 2003.

Coetzee , J. m. Tierras de poniente. Trad. Javier Calvo. Barcelona: Random House Mondadori, 2009.

h Ayes, PAtrick .J. M. Coetzee and the Novel. Oxford: University of Oxford Press, ebook, loc. 408, 2010.

h uchteon , I inDA . A Poetics of Postmodernism: History, Theory, Fiction. Nueva York: Routledge, 1988.

$\mathrm{n}$ Agel , $\mathrm{t}$ homAS. The View from Nowhere. Oxford: Oxford University Press, 1986.

Pippin, $r$ obert . "The Paradoxes of Power in the Early Novels of J. M. Coetzee", en J. M. Coetzee and Ethics. Nueva York: Columbia University Press, 2010.

Shklovsky , viktor . Literature and Cinematography. Londres: Dalkey Archive, 2008.

StAm , $r$ obert . Literature through Film: Realism, Magic and the Art of Adaptation. Nueva York: Blackwell Publishing, 2005.

$\mathrm{y}$ eoh, $\mathrm{g}$ ilbert . "J. M. Coetze and Samuel Beckett: Nothingness, Minimalism and Indeterminacy", en Ariel: A Review of International English Literature, vol. 31 núm. 4 (octubre 2000).

mente puedo dar una respuesta: capturar, encerrar, mantener. Pero, ¿hasta qué punto es real nuestra posesión?" (Coetzee 2003: 156). 